\section{Description of vaccination in dogs, cats and rabbits attending veterinary practices in the UK through use of e-health records}

\section{Ana Muniesa1, Alan D Radford², David Singleton ${ }^{2}$, Susan Dawson ${ }^{3}$, Rosalind Gaskell $^{2}$, Bethaney Heayns ${ }^{2}$, Philip H Jones $^{2}$, Tarek Menacere ${ }^{2}$, Peter J Noble ${ }^{3}$, Fernando Sánchez-Vizcaíno ${ }^{2}$ \\ 1 Universidad de Zaragoza, Facultad de Veterinaria, Zaragoza, Spain 2 University of Liverpool, Institute of Infection and Global Health, Neston, UK \\ 3 University of Liverpool, School of Veterinary Science, Neston, UK}

Vaccination forms a major part of the veterinary professions' strategy to improve the health and welfare of companion animals. However, there is a lack of national information regarding vaccination in small animal practice. Here we used data gathered through the Small Animal Veterinary Surveillance Network (SAVSNET), to provide new insight into vaccination behaviour in companion animals' healthcare settings in the UK.

Data were collected electronically in near real-time from 101 veterinary practices (245 premises). These data comprised animal signalment, owner's full postcode and the vaccination histories of 77,981 dogs, 34,930 cats and 3,835 rabbits included within the SAVSNET database between 22/11/2013 and 30/09/2015. Vaccination dates ranged between 24/03/1998 and 30/09/2015. The term 'vaccinated' was defined as any animal that had received at least one vaccination and it was recorded in its clinical health records. In dogs, pathogens considered a 'core' vaccines were canine distemper virus (CDV), canine parvovirus (CPV) and canine hepatitis virus (CHV); in cats, feline calicivirus (FCV), feline herpesvirus (FHV) and feline panleucopenia (FPV); and in rabbits, vaccines for myxomatosis and rabbit haemorrhagic disease (RHD). Proportions and confidence intervals (95\%) were calculated using robust standard errors to allow for the clustering within veterinary practices.

Overall, $77.9 \pm 0.2 \%$ of all animals were vaccinated $(81.5 \pm 0.3 \%$ of dogs, $73.1 \pm 0.5 \%$ of cats and $48.4 \pm 1.6 \%$ of rabbits). When animals of less than six months of age were considered, $76.7 \pm 0.5 \%$ of animals were vaccinated $(82.1 \pm 0.6 \%$ of dogs, $69.1 \pm 1.0 \%$ of cats and $45.3 \pm 3.2 \%$ of rabbits). Median age (years) at first vaccination was $0.21 \pm 0.01$ for dogs, $0.33 \pm 0.1$ for cats and $0.35 \pm 0.3$ for rabbits. More than half $(\sim 62.0 \%)$ of all cats were vaccinated for each core pathogen, whilst only $\sim 46.0 \%$ of dogs were vaccinated against each core pathogen. A total of $46.9 \%$ and $46.7 \%$ of rabbits were vaccinated for myxomatosis and $\mathrm{RHD}$, respectively. Canine non-core vaccines such as vaccines for Leptospira spp., and parainfluenza virus were always administered with the core vaccines. In cats, the same was true for the non-core vaccine against feline leukemia virus.

A considerable proportion of dogs, cats and rabbits had not been vaccinated against the core pathogens. In dogs and cats, few of the traditionally considered as non-core vaccines were always administered along with core-vaccines indicating the need of further research in the area of companion animal vaccinology to generate optimal recommendations for its vaccination.

\section{Prevalence of antimicrobial resistance amongst canine Pseudomonas aeruginosa clinical isolates between 2011-2015}

\section{Vanessa Schmidt, Lucy Botham, Laura Buckley, Dorina Timofte \\ University of Liverpool, Neston, UK}

Pseudomonas aeruginosa is commonly implicated in a variety of bacterial infections in dogs and is of veterinary concern due to broad-spectrum inherent antimicrobial resistance and its ability to acquire further resistance to key antimicrobials. This study aimed to investigate the prevalence of antimicrobial resistance (AMR) amongst canine $P$. aeruginosa isolates $(n=113)$ during the last five years. Data from a UK basedMicrobiology Diagnostics Laboratory was collected from 2011 until 2015. Antimicrobial resistance was determined by minimum inhibitory concentration (MIC; CLSI 2008) and AMR to six antimicrobials potentially effective against $P$. aeruginosa (enrofloxacin, marbofloxacin, gentamicin, amikacin, ticarcillin and ticarcillin-clavulanate), was investigated. The prevalence of AMR to each antimicrobial for each year and overall was calculated with $95 \%$ confidence intervals. In addition, the prevalence of AMR to each antimicrobial was calculated according to site of isolation (otitis, skin and soft tissue infections (SSTI), urinary tract or other infections). The majority of isolates were resistant to enrofloxacin (81\%; 95\% Cl: 73-88), followed by marbofloxacin (43\%; 95\% Cl: $26-44)$ and then gentamicin (24\%; 95\% Cl: 17-33). Resistance to ticarcillin with (8\%; 95\% Cl: $4-15)$ or without amoxicillin-clavulanate (12\%; 95\% Cl: 7-19) was uncommon whilst resistance to amikacin (2\%; 95\% Cl: 1-6) was rare. AMR the investigated antimicrobials had not significantly increased between 2011 and 2015. Isolates from otitis and SSTI were more likely to be resistant to enrofloxacin and marbofloxacin compared to isolates from urinary tract isolates. While isolates from otitis were more likely to be resistant to gentamicin compared to isolates from SSTI, and this resistance was not detected amongst urinary tract isolates. The two isolates that were amikacin resistant were both detected from otitis cases. These findings suggest that AMR amongst canine Pseudomonas isolates, particularly isolates from otitis cases, is highest against antimicrobials authorised for the treatment of Gram-negative infections in dogs, highlighting the need for prudent antimicrobial use. 